\title{
Article \\ Response of Wheat DREB Transcription Factor to Osmotic Stress Based on DNA Methylation
}

\author{
Huihui Wang ${ }^{1,+}$, Yanqiu Zhu ${ }^{1,+}{ }^{+}$Ping Yuan ${ }^{1}$, Shanglin Song ${ }^{1}$, Tianyu Dong ${ }^{1}$, Peilei Chen ${ }^{1}$, Zhikun Duan ${ }^{2}$, \\ Lina Jiang ${ }^{1}$, Longdou Lu ${ }^{1, *}$ and Hongying Duan ${ }^{1, *}$ \\ 1 College of Life Sciences, Henan Normal University, Xinxiang 453007, China; hui15690788798@163.com (H.W.); \\ Qiqt536@163.com (Y.Z.); yp15249710089@163.com (P.Y.); dxdhy@163.com (S.S.); \\ tianyuwithchen@163.com (T.D.); Chenp1536@163.com (P.C.); 041139@htu.edu.cn (L.J.) \\ 2 College of Life Sciences, Henan University, Kaifeng 475004, China; duanzhikun@henu.edu.cn \\ * Correspondence: 041079@htu.edu.cn (L.L.); dxdhy@126.com (H.D.) \\ + These authors contributed equally to this work.
}

check for

updates

Citation: Wang, H.; Zhu, Y.; Yuan, P.; Song, S.; Dong, T.; Chen, P.; Duan, Z.; Jiang, L.; Lu, L.; Duan, H. Response of Wheat DREB Transcription Factor to Osmotic Stress Based on DNA Methylation. Int. J. Mol. Sci. 2021, 22, 7670. https://doi.org/10.3390/ ijms22147670

Academic Editors: Giampiero Cai, Luigi Parrotta and Lavinia Mareri

Received: 30 June 2021

Accepted: 16 July 2021

Published: 18 July 2021

Publisher's Note: MDPI stays neutral with regard to jurisdictional claims in published maps and institutional affiliations.

Copyright: (c) 2021 by the authors. Licensee MDPI, Basel, Switzerland. This article is an open access article distributed under the terms and conditions of the Creative Commons Attribution (CC BY) license (https:// creativecommons.org/licenses/by/ $4.0 /)$.

\begin{abstract}
Dehydration-responsive element-binding protein (DREB) plays an important role in response to osmotic stress. In this study, DREB2, DREB6 and Wdreb2 are isolated from wheat AK58, yet they belong to different types of DREB transcription factors. Under osmotic stress, the transcript expression of DREB2, DREB6 and Wdreb2 has tissue specificity and is generally higher in leaves, but their expression trends are different along with the increase of osmotic stress. Furthermore, some elements related to stresses are found in their promoters, promoters of DREB2 and Wdreb2 are slightly methylated, but DREB6's promoter is moderately methylated. Compared with the control, the level of promoter methylation in Wdreb2 is significantly lower under osmotic stress and is also lower at CG site in DREB2, yet is significantly higher at CHG and CHH sites in DREB2, which is also found at a CHG site in DREB6. The status of promoter methylation in DREB2, DREB6 and Wdreb2 also undergoes significant changes under osmotic stress; further analysis showed that promoter methylation of Wdreb2 is negatively correlated with their expression. Therefore, the results of this research suggest the different functions of DREB2, DREB6 and Wdreb2 in response to osmotic stress and demonstrate the effects of promoter methylation on the expression regulation of Wdreb2.
\end{abstract}

Keywords: Triticum aestivum; osmotic stress; DREB transcription factor; promoter; DNA methylation

\section{Introduction}

Osmotic stress is one of the major abiotic stresses, which not only affects the growth and development of plants, but also severely restricts the sustainable production of agricultural biomass [1]. In order to adapt or resist to adverse environments, plants usually respond morphologically, physiologically and molecularly, such as by regulating the expression of stress resistance genes [2-5]. Plant resistances are generally controlled by multiple genes, it is well known that one transcription factor can regulate the expression of multiple functionally related genes, and many transcription factors are related to resistant response in plants, such as DREB, bZIP, MYB and WRKY [6].

DREB transcription factor belongs to AP2/EREBP family and contains one AP2/EREBP domain which is composed of about 60 amino acid residues with the conserved element YRG and RAYD [7]. Through the AP2/EREBP domain, DREB transcription factor could specifically bind to the dehydration responsive element/C-repeat (DRE/CRT) (core sequence: $5^{\prime}$-CCGAC-3'). This binding is involved in regulating the expression of genes related with abiotic stress response to high salt, low temperature or drought, which would enhance the resistances of plants to adverse stress [6]. Arabidopsis DREB1/CBF can regulate the expression of $r d 29 A$, erd10, cor6.6, cor15a, $r d 17$ and other stress-resistant genes to drought, low temperature and so on [8]. Overexpression of DREB1A in transgenic Arabidopsis also could enhance the expression of its downstream target genes, which increased the 
drought tolerance of transgenic Arabidopsis significantly [9]. So far, many genes encoding DREB transcription factor have been identified from Arabidopsis, Maize, Soybean, Sesame, etc.; their expression could be induced and would accumulate rapidly in a short time under abiotic stress [10]. However, the mechanisms of the regulation of DREB gene expression are less studied, especially the epigenetic regulations.

DNA methylation is a major epigenetic modification and has a vital function in the growth and development of plants [11]. DNA methylation in plants is easily affected by physiological status, developmental stage and environmental factors [12]. Under osmotic stress, the state of DNA methylation altered in plants, and methylation level and patterns were significantly different [13]. Fan et al. found that the methylation level of Dendrobium huoshanense decreased, and methylation polymorphism gradually increased along with the increase of osmotic stress [14]. The methylation level of Ryegrass also decreased under osmotic stress, and the expression of demethylated related genes was up-regulated [15]. Some studies indicated that the improved resistance of plants to stresses is connected with the involvement of DNA methylation in regulating the expression of stress-resistant genes [16]. For example, the physiological processes of Rice are related to DNA methylation in response to osmotic stress [13], the modification of DNA methylation status is closely connected with drought resistance of Fraxinus hybrid trees [17]. Furthermore, methylation or demethylation of genes in plants can cause the difference of gene expression under osmotic stress [18], which would result in drought escape or tolerance [19].

Wheat (Triticum aestivum L.), belonging to Gramineae family, is rich in starch, protein, sugar and other substances, and is one of the main food crops. In recent years, osmotic stress is a significant reason for the restriction of sustainable increase of wheat production [20]. However, studies on the response of wheat to osmotic stress are usually confined to phenotype, structure, physiology and biochemistry, or stress-resistant genes [21]. The epigenetic regulation of wheat response to osmotic stress is rarely involved, especially the regulatory role of DNA methylation in DREB transcription factor under osmotic stress. In this study, three members of the DREB family in wheat AK58 are identified, and the expression and promoter methylation of DREB genes are analyzed under osmotic stress. Thus, this investigation is beneficial to explore the regulatory mechanism of DNA methylation in the response of plants to osmotic stress.

\section{Results}

\subsection{Amplification and Sequence Analysis of DREBs}

As shown in Figure S1, CDS sequences of DREB2, DREB6 and Wdreb2 in wheat AK58 comprise $732 \mathrm{bp}, 837 \mathrm{bp}$ and $1035 \mathrm{bp}$ respectively; DREB2 and Wdreb2 have no intron, but one 712-bp intron is found in DREB6. A typical AP2/EREBP domain is identified in the deduced protein sequences of three DREB genes (Figure S1). AP2/EREBP domain contains YRG and RAYD conserved modules with three $\beta$ folds and one $\alpha$ helix, valine (V) and glutamate (E) are highly conserved at 14 th or 19 th residue of AP2/EREBP domain (Figure S2a). The amino acid sequences of DREB2, DREB6 and Wdreb2 are further compared and analyzed, and despite the low overall sequence similarity among three DREBs (33.24\% identity) (Figure S2b), their AP2/EREBP domains have $73.25 \%$ identity, even up to $83.93 \%$ between DREB6 and Wdreb2 (Figure S2).

The deduced protein sequences of DREB2, DREB6 and Wdreb2 from wheat AK58 are also aligned with their homologous sequences (Figure S3). The sequence identity between DREB2 and Aegilops tauschii ERF is 95\%, but has only around 60\% identity with other homologous sequences (Table S1). As listed in Table S1, DREB6 has higher similarity to some homologous sequences (approximately $97 \%$ identity), the higher sequence similarity is observed between Wdreb2 and Aegilops tauschii DREB2B, Aegilops speltoides DREB1, Triticum turgidum DRF, Triticum dicoccoides DREB or Triticum urartu DREB2B (Table S1). 


\subsection{The Expression Patterns of DREBs in Wheat}

As shown in Figure 1a, the expression level of DREB2 in leaves is significantly higher than that in roots under osmotic stress, similarly in DREB6 as stressed for $2-10 \mathrm{~h}$ (Figure $1 \mathrm{~b}$ ). Compared with the level in roots, the expression level of Wdreb2 in leaves is also significantly higher except for the stress of $4 \mathrm{~h}$ or $10 \mathrm{~h}$ (Figure 1c). Furthermore, the expression of DREB2, DREB6 and Wdreb2 is different under osmotic stress and has its own unique expression profile (Figure 1).

(a)

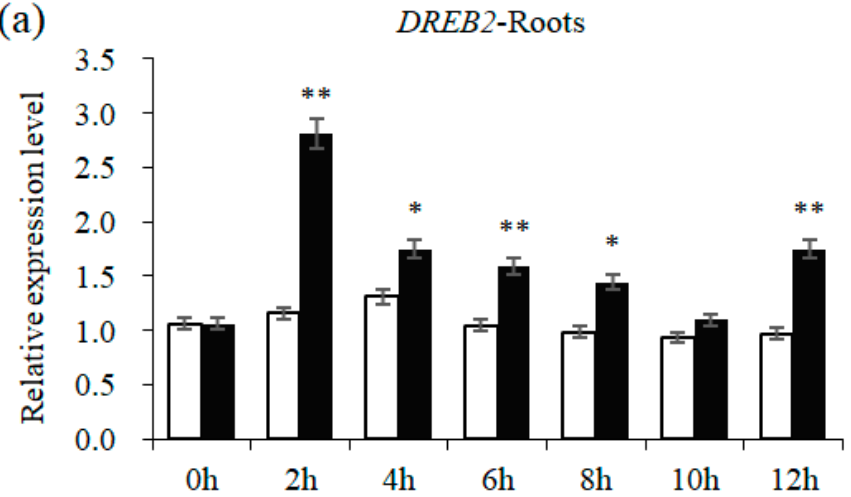

(b)

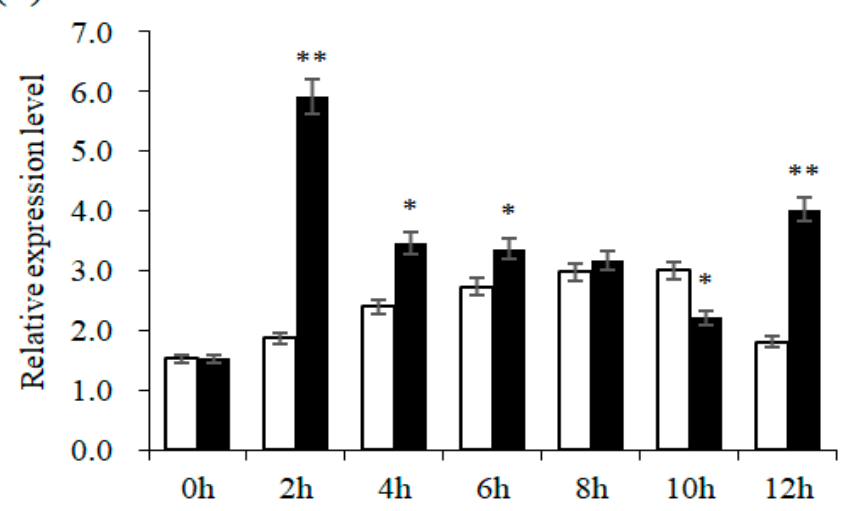

(c)

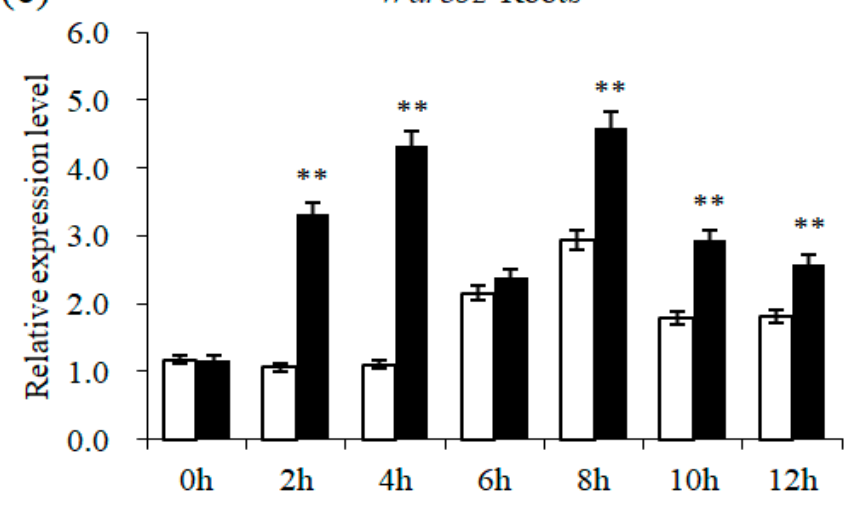

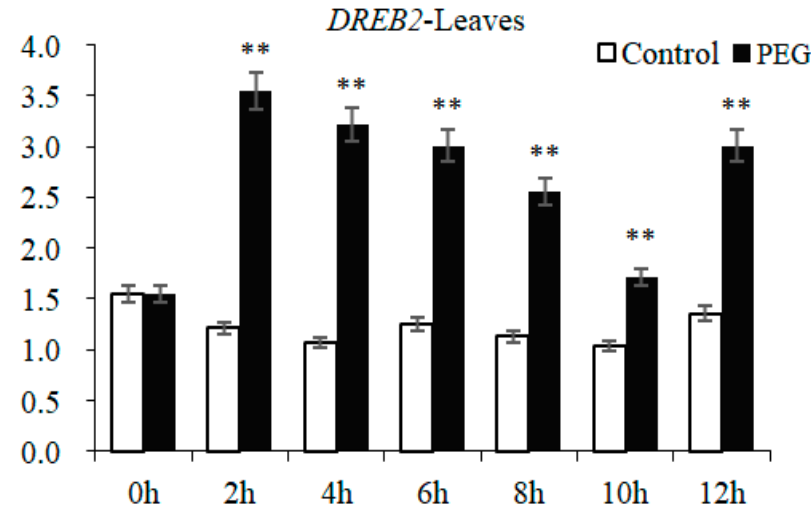

DREB6-Leaves

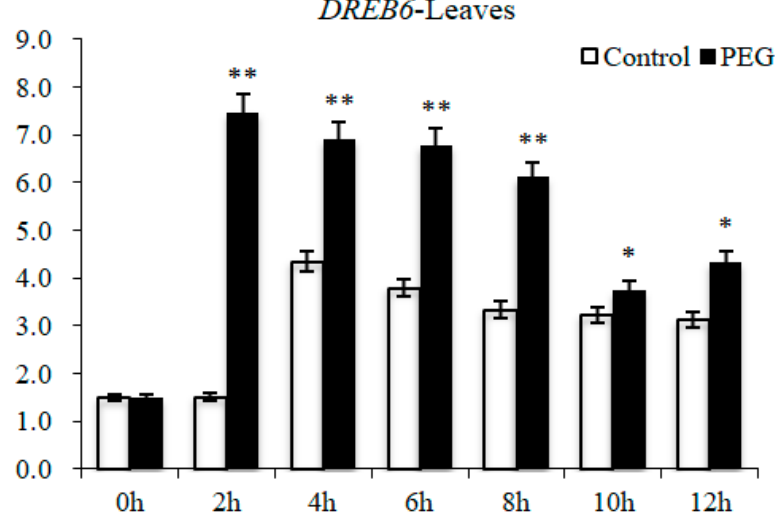

Wdreb2-Leaves

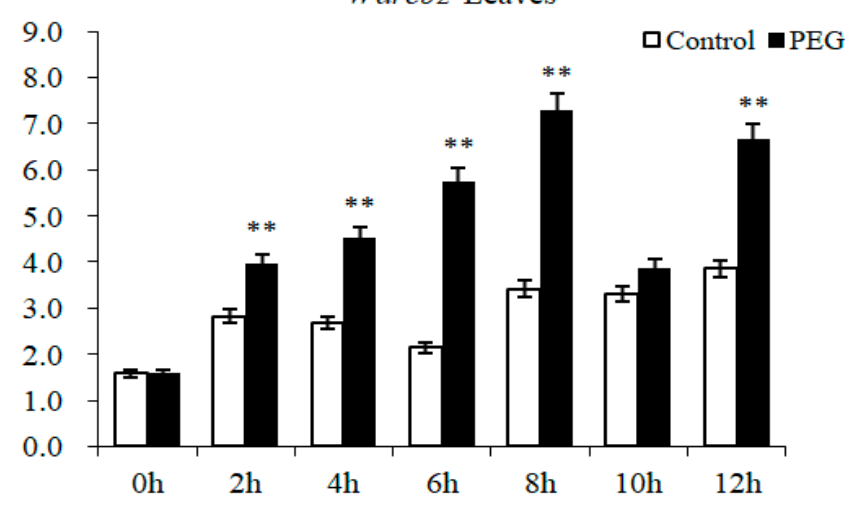

Figure 1. The expression analysis of wheat $D R E B$ genes under osmotic stress. Quantitative real-time PCR (qRT-PCR) analysis of DREB2, DREB6 and Wdreb2 expression are shown in (a-c); the transcript abundances of three DREBs are analyzed in wheat seedlings treated with $15 \%$ PEG6000 solution. The error bar is the standard error of the mean. * and ** represent significant difference relative to the control conditions at the levels of 0.05 and 0.01 , respectively. 
Under osmotic stress, the expression of DREB2 displays similar trends in roots and leaves (Figure 1a). DREB2 transcript abundance would increase to a higher level after stressed for $2 \mathrm{~h}(p<0.01)$, then declines, and is lower after stressed for $10 \mathrm{~h}$, which is still higher in roots and leaves than the control and is especially significant in leaves $(p<0.01)$. The expression trends of DREB6 are also similar in roots and leaves under osmotic stress (Figure 1b). A significant rise in the DREB6 transcript level is also observed after stressed for $2 \mathrm{~h}(p<0.01)$, although DREB6 subsequently exhibits the declined expression, which is significantly higher in leaves than the control (Figure 1b). As shown in Figure 1c, under osmotic stress, Wdreb2 is up-regulated in roots except for the stress of $6 \mathrm{~h}$, especially after stressed for $2-4 \mathrm{~h}(p<0.01)$. Compared with the control, except for the expression stressed for $10 \mathrm{~h}, \mathrm{Wdreb} 2$ transcript level in leaves also increases significantly under osmotic stress, especially after stressed for $6-8 \mathrm{~h}(p<0.01)$.

\subsection{Promoter Analysis of Wheat DREB Genes}

In this study, the promoters of DREB2, DREB6 and Wdreb2 are isolated and submitted to GenBank (MT974473: 1735 bp, MT974471: 1792 bp, MT974472: 649 bp). As shown in Figure S4 and Tables S2-S4, the promoters of wheat DREB genes contain basic regulatory elements, such as TATA-box and CAAT-box. There are 13, 10 and 5 TATA-boxes in the promoters of DREB2, DREB6 and Wdreb2, respectively. Many elements related to stresses are also found in the promoters of DREB2, DREB6 and Wdreb2 such as drought response element DRE/CRT, low temperature response element LTR, abscisic acid response element ABRE, light response element GAG-motif, drought-induced element MYB binding sites, etc. (Figure S4, Tables S2-S4).

Further analysis showed that there are some unique elements in the promoters of $D R E B 2, D R E B 6$ and Wdreb2. For example, light response element MNF, leaf development element HD-ZIP and meristem specificity element OCT are specifically present in the promoter of DREB2 (Table S2). A series of specific functional elements are also identified in the promoter of DREB6, such as ethylene response element ERE, fungal elicitor response element W-box and MeJA regulatory element CGTCA-motif (Table S3). Moreover, root specificity elements as1, zein metabolism regulation element $\mathrm{O} 2$-site, light response element C-box, and CE3 element involved in ABA and VP1 reactions are detected in the promoter of Wdreb2 (Table S4).

\subsection{Promoter Methylation Analysis of DREB Genes}

The distribution of $\mathrm{CpG}$ island in the promoter regions of whet $D R E B s$ are analyzed. It is found that one CpG island is present in the promoter of DREB2, DREB6 or Wdreb2, and its length is $234 \mathrm{bp}, 436 \mathrm{bp}$ and $559 \mathrm{bp}$, respectively (Figure S5). These putative CpG islands are preceded by some functional elements such as the abscisic acid responsive element, light responsive element, low-temperature responsive element and so on (Figure S4, Tables S2-S4).

Some $\mathrm{CpG}$ island regions with a higher CG percentage are further identified in wheat leaves (Figure S6). The majority of methylation sites are in $\mathrm{CHH}$ context in the promoter regions of $D R E B 2, D R E B 6$ and Wdreb2, but DNA methylation has a strong preference for CG context (Table 1). In the promoter region of DREB2, methylation in CHH site is not detected and methylation rates of $\mathrm{CG}$ and $\mathrm{CHH}$ sites are $2.38 \%$ and $1.03 \%$, belonging to mild methylation (Figure S6a; Table 1). Figure S6b and Table 1 shows dense methylation at CG site $(88.08 \%)$, moderate methylation at CHG site (51.36\%) and mild methylation at CHH site (4.93\%) in the promoter region of DREB6. In the promoter region of Wdreb2, methylation rates of CG, CHG and CHH sites are $1.89 \%, 1.0 \%$ and $0.29 \%$, respectively, which are all mildly methylated (Figure S6c; Table 1). 
Table 1. Methylation analysis of promoter regions in wheat DREB genes.

\begin{tabular}{ccccc}
\hline Gene & Pattern & $\begin{array}{c}\text { Pattern Frequency } \\
\mathbf{( \% )}\end{array}$ & $\begin{array}{c}\text { Methylation Rate } \\
\mathbf{( \% )}\end{array}$ & $\begin{array}{c}\text { Total Methylation Rate } \\
\mathbf{( \% )}\end{array}$ \\
\hline \multirow{2}{*}{ DREB2 } & CG & 19.09 & 2.38 & \\
& CHG & 11.82 & 0.00 & \\
& CHH & 69.09 & 1.03 & \\
DREB6 & CG & 25.93 & 88.08 & 31.89 \\
& CHG & 11.11 & 51.36 & \\
& CHH & 62.96 & 4.93 & 0.88 \\
& CG & 29.60 & 1.89 & \\
& CHG & 16.00 & 1.00 & \\
& CHH & 54.40 & 0.29 & \\
\hline
\end{tabular}

\subsection{Methylation Level of DREB Promoters under Osmotic Stress}

Under osmotic stress, cytosine methylation would alter in the promoter regions of DREB2, DREB6 and Wdreb2 from wheat leaves (Figure 2). Compared with the control, methylation rate at CG site in the promoter region of $D R E B 2$ decreases obviously under osmotic stress $(p<0.01)$, but methylation rates at $\mathrm{CHG}$ and $\mathrm{CHH}$ sites increase significantly, especially in CHG site $(p<0.01)$ (Figure 3a).

(a)

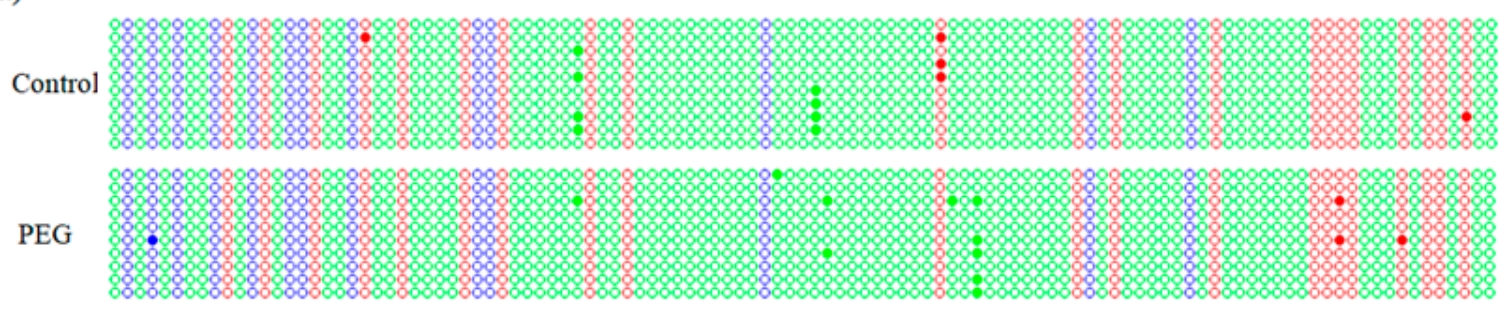

(b)

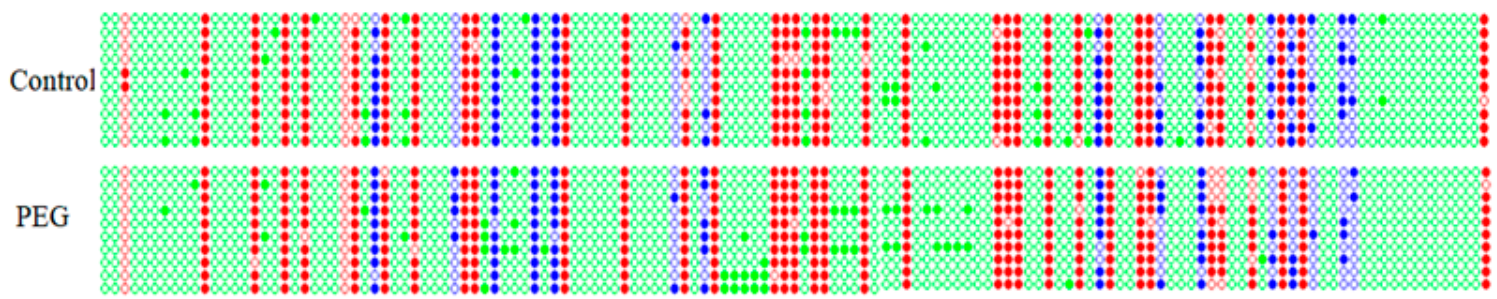

(c)

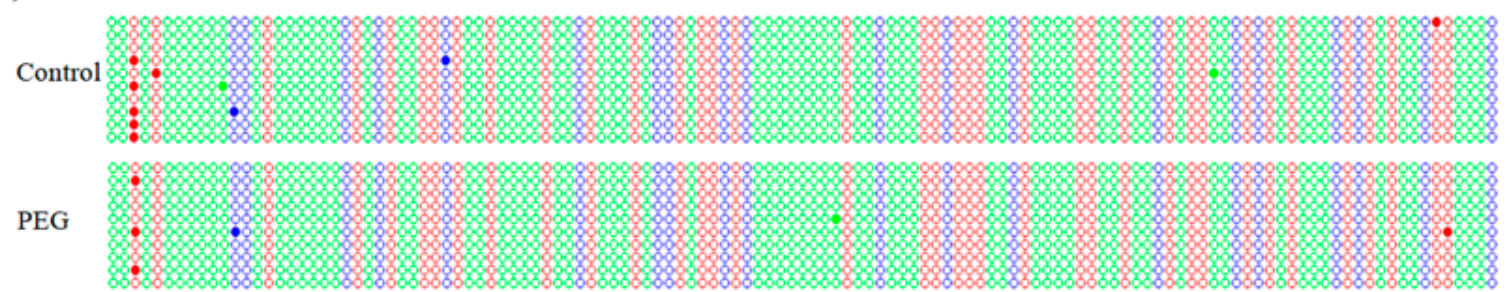

Figure 2. Methylation profiles in the promoter regions of wheat $D R E B$ genes under osmotic stress. $(\mathbf{a}-\mathbf{c})$ represent cytosine methylation maps for the promoter sequences of DREB2, DREB6 and Wdreb2 in wheat leaves stressed with 15\% PEG6000 solution for $12 \mathrm{~h}$. Red, blue and green circles represent CG, CHG or CHH. Filled and hollow circles denote methylated and unmethylated cytosine, respectively. Each row represents the sequencing result of one positive clone. 
(a)

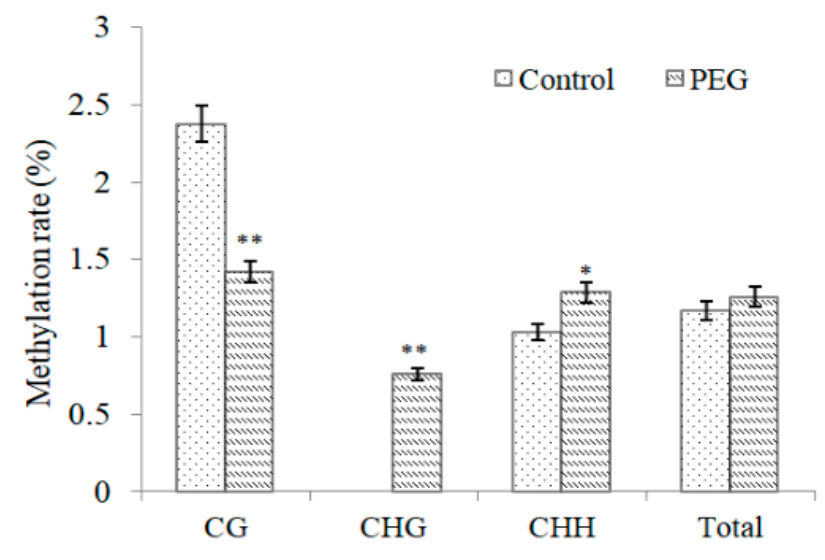

(b)

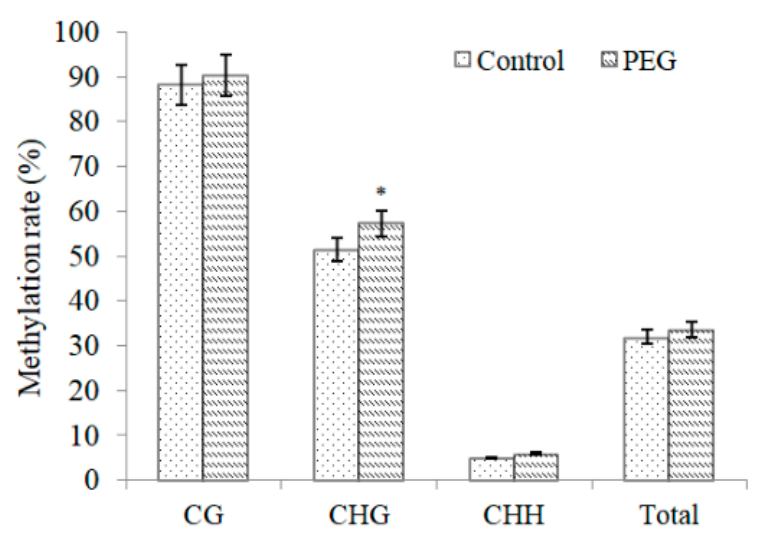

(c)

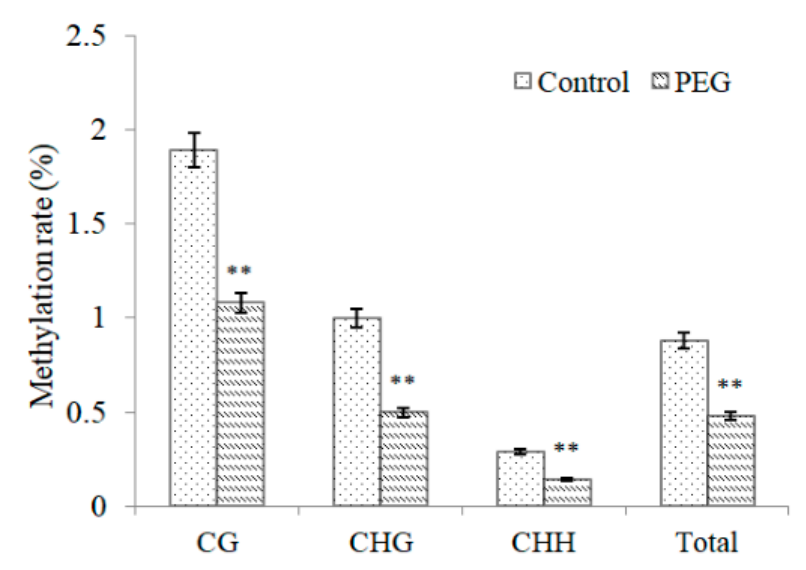

Figure 3. Effect of osmotic stress on methylation level of DREB promoters in wheat. $(\mathbf{a}-\mathbf{c})$ represent methylation rates for the promoter regions of DREB2, DREB6 and Wdreb2 in wheat leaves stressed with $15 \%$ PEG6000 solution for $12 \mathrm{~h}$. The error bar is the standard error of the mean. * and ${ }^{* *}$ represent significant difference relative to the control conditions at the levels of 0.05 and 0.01 , respectively.

As shown in Figure 3b, under osmotic stress, the increased methylation level of DREB6 promoter is observed, which only occurrs at CHG site $(p<0.05)$. Figure $3 c$ displays the changes in promoter methylation of Wdreb2 under osmotic stress. Compared with the control, the overall methylation level was significantly lower $(p<0.01)$; methylation rates at CG, CHG and CHH sites also significantly declines under osmotic stress $(p<0.01)$.

\subsection{Methylation Status in DREB Promoters under Osmotic Stress}

As listed in Table 2, methylation status in the promoter regions of DREB2, DREB6 and Wdreb2 alters significantly under osmotic stress for $12 \mathrm{~h}$. The demethylation at $3 \mathrm{CG}$ sites and $1 \mathrm{CHH}$ site are detected in the promoter of $D R E B 2$, and the hypermethylation of $D R E B 2$ promoter at $\mathrm{CG}, \mathrm{CHG}$ and $\mathrm{CHH}$ sites is also found, being 2, 1 and 3, respectively. 
Table 2. Methylation patterns in promoter regions of wheat DREBs under osmotic stress.

\begin{tabular}{lcccc}
\hline \multirow{2}{*}{ Gene } & Cytosine Type & No. of Cytosine & \multicolumn{2}{c}{ No. of Methylation Site } \\
\cline { 4 - 5 } DREB2 & CG & 21 & Hypermethylation Site & Demethylation Site \\
\hline \multirow{5}{*}{ DREB6 } & CHG & 13 & 2 & 3 \\
& CHH & 76 & 1 & 0 \\
& CG & 34 & 3 & 1 \\
\multirow{3}{*}{ Wdreb2 } & CHG & 15 & 1 & 1 \\
& CG & 84 & 1 & 8 \\
& CHG & 37 & 10 & 2 \\
& CHH & 20 & 1 & 2 \\
\hline
\end{tabular}

Under osmotic stress, $10 \mathrm{CHH}$ sites, $1 \mathrm{CG}$ site and $1 \mathrm{CHG}$ site are hypermethylated in the promoter of DREB6. $8 \mathrm{CHH}$ sites, 1 CG site and $1 \mathrm{CHG}$ site are demethylated (Table 2). Compared to CHG sites, the methylation status of CHH and CG sites in Wdreb2 promoter are affected strongly by osmotic stress (Table 2): for example, $1 \mathrm{CHH}$ site and $1 \mathrm{CG}$ site are hypermethylated, whereas $2 \mathrm{CHH}$ sites and 2 CG sites are demethylated.

\subsection{Correlation Analysis between Promoter Methylation and Expression of DREBS}

In order to explore the correlation between promoter methylation and expression of wheat DREBs under osmotic stress, the relative expression levels of DREBs in wheat leaves and methylation rates at CG, $\mathrm{CHG}$ or $\mathrm{CHH}$ sites in their promoter regions were respectively analyzed by Pearson correlation coefficient. As listed in Table S5, Pearson coefficient $\mathrm{r}$ between the expression level of $\mathrm{Wdreb2}$ and methylation rate at CG, CHG and $\mathrm{CHH}$ sites is respectively $-0.986,-0.973$ and -0.878 , indicating that a significant negative correlation exists between promoter methylation and the gene expression of Wdreb2. Although significant negative correlation exists between the gene expression of $D R E B 2$ or DREB6 and the methylation rate of CG (Table S5), promoter methylation of $D R E B 2$ or DREB6 has no negative correlation with its expression under osmotic stress.

\section{Discussion}

DREB transcription factor can specifically bind to DRE/CRT elements in the promoter of stress-responsive genes and can enhance the response or tolerance of plants to adverse environmental conditions [6]. In this study, DREB2, DREB6 and Wdreb2 are isolated from wheat AK58, and one 712-bp intron is found in DREB6, AP2/EREBP domains of DREB2, DREB6 and Wdreb2 have 73.25\% identity; the amino acids at the 14th and 19th of AP2/EREBP domain are conservatively V and E, respectively. However, the similarity is lower among the full-length nucleotide sequences or amino acid sequences of $D R E B 2$, DREB6 and Wdreb2. BLASTP analysis further revealed that DREB2, DREB6 and Wdreb2 are different types of DREB transcription factors and might respectively belong to DREBA-4 class, DREB-2 class and DREB-1 class [22,23].

Under abiotic stresses such as drought, low temperature, high salt, etc., the expression of DREB transcription factor can change [24,25]. In this study, the expression of DREB2, $D R E B 6$ and Wdreb2 also alters under osmotic stress and generally accumulates to the higher level after stressed for $2 \mathrm{~h}$, but they have different expression trends along with the increase of stress time. The expression levels of wheat $D R E B s$ are also different, which is similar to other research [26]. Further analysis showed that the expression of wheat DREBs has tissue specificity, and the accumulation of DREBs transcripts is obviously higher in leaves, which is also found in other research [27]. The expression of DREB in Daucus carota showed tissue specificity as well, and the main role of DCDREB-A1-1 and DCDREB-A1-2 is in leaves and roots, respectively [28].

It is well known that the cis-acting regulatory elements in the promoters provide the possibility for the transcription and expression of genes [29], and there are some cis-acting 
elements related to stresses, such as DRE/CRT, ERE, ABRE, LTR and so on [30]. Except typical regulatory elements TATA-box and CAAT-box, the promoters of DREB2, DREB6 and Wdreb2 in wheat AK58 contain DRE/CRT, LTR, ABRE, drought-induced MYB binding site, etc., which demonstrates that the expression of DREB2, DREB6 and Wdreb2 may be influenced by adverse environmental factors. Some studies found that DNA methylation can regulate the expression of stress-responsive genes and play an important role in the response of plants to adverse stress [16], especially promoter methylation which has more significant effects on gene expression [31]. In the promoter regions of DREB2, DREB6 and Wdreb2, CpG islands with many cis-acting elements are detected. BSP analysis showed that there are more $\mathrm{CHH}$ sites and less $\mathrm{CHG}$ sites in the promoter regions of wheat $D R E B s$, but the methylation rates at CG sites are the highest.

Furthermore, the degree and state of DNA methylation in plants can change under drought stress, low temperature, high salt and other conditions [32,33], especially the change of methylation state in the promoter of some genes [34]. In this study, methylation level also alters in the promoter regions of DREBs under osmotic stress. Compared with the control, the level of promoter methylation in Wdreb2 is significantly lower under osmotic stress and is also lower at the CG site in DREB2, yet is significantly higher at CHG and CHH sites in DREB2, which is also found at the CHG site in DREB6. In addition, methylation status in the promoter regions of wheat DREBs undergoes a significant change under osmotic stress, such as demethylation and hypermethylation. Zilberman also found that gene expression can be promoted or inhibited by the demethylation and hypermethylation of promoter, respectively [35].

Further analysis showed that promoter methylation of Wdreb2 is negatively correlated with their expression, which is also found in other studies $[35,36]$. Although the promoters of DREB2 and Wdreb2 are both slightly methylated, the expression of Wdreb2 was significantly higher than that of DREB2, indicating that promoter methylation might have little effect on the gene expression of DREB2 and its promoter possibly belongs to a low CpG-containing promoter. Similarly, the promoters of z1B4 and z1B6 in Zea mays are almost not methylated [37]; DNA methylation is also not found in the promoters of some genes in Arabidopsis or tomato and only occurs in their coding regions [38,39]. In addition, one CpG island is also found in the coding region of wheat DREBs, and the CpG island almost covers the whole coding region of DREB2. However, the relationship between DNA methylation in the coding region and gene expression of wheat $D R E B$ genes is unclear; the mechanism of DNA methylation regulating the expression of wheat DREB genes therefore needs to be further studied.

\section{Materials and Methods}

\subsection{Cultivation and Treatment of Wheat Seedlings}

In this study, seeds of wheat AK58 are kindly provided by Xinxiang Academy of Agricultural Science, Xinxiang, Henan, China. Cultivation of wheat seedlings is performed according to Duan et al. [33], wheat seeds are firstly surface-sterilized for $10 \mathrm{~min}$ by $0.1 \%$ $\mathrm{HgCl}_{2}$ and then are washed for $50 \mathrm{~min}$ in sterile water. Subsequently, sterilized seeds are sown in pots (with a diameter of $15 \mathrm{~cm}$ ) containing nutrition soil and vermiculite (1:1), cultured at $24 \pm 1{ }^{\circ} \mathrm{C}$ with $45 \%$ relative humidity in a $14 \mathrm{~h}$ photoperiod of $50 \mu \mathrm{mol} \mathrm{m}{ }^{-2} \mathrm{~s}^{-1}$ light intensity, and finally are irrigated with $5 \mathrm{~mL}$ distilled water every two days.

At the three-leaf stage, wheat seedlings are exposed to 15\% PEG6000 solution for $2 \mathrm{~h}$, $4 \mathrm{~h}, 6 \mathrm{~h}, 8 \mathrm{~h}, 10 \mathrm{~h}$ and $12 \mathrm{~h}$. Roots and leaves of PEG6000-treated and untreated wheat seedlings are collected, immediately frozen with liquid nitrogen and then stored at $-80^{\circ} \mathrm{C}$.

\subsection{Extraction of Genomic DNA}

Genomic DNA is extracted from roots or leaves of wheat seedlings by the cetyltriethyl ammonium bromide (CTAB) method [40]. The yield and purity of genomic DNA are determined at $260 \mathrm{~nm}$ with micro-spectrophotometry, and the integrity of genomic DNA 
is detected by $0.8 \%$ agarose gel electrophoresis. Subsequently, genomic DNA from wheat seedlings is stored at $-20^{\circ} \mathrm{C}$.

\subsection{Isolation and Reverse Transcription of RNA}

Total RNA from roots or leaves of wheat seedlings is extracted using RNAiso Plus kit (TaKaRa, Kyoto, Japan) according to the instructions. DNase treatment and phenolchloroform extraction are performed to remove DNA, and RNA samples are dissolved in RNase-free $\mathrm{ddH}_{2} \mathrm{O}$. The integrity of total RNA is verified by $1.0 \%$ agarose gel electrophoresis, and the yield and purity of total RNA are determined with UV spectrophotometer. RNA samples are used for the generation of cDNA or stored at $-80^{\circ} \mathrm{C}$. First strand cDNA is synthesized with the HiScript II Q RT SuperMix for qPCR (+gDNA wiper) kit (Vazyme, Nanjing, China).

\subsection{Amplification and Bioinformatic Analysis of DREB Genes}

In order to isolate $D R E B$ genes from wheat $A K 58$, specific primers are designed according to the sequences of wheat DREB2 (GU785008), DREB6 (AY781361) and Wdreb2 (AB193608), and are listed in Table S6. Genomic DNA and cDNA of wheat AK58 were used as the amplification templates to obtain DNA or CDNA sequences of DREB genes.

In this experiment, a PCR reaction system is composed of a $2.0 \mu \mathrm{L}$ DNA template,

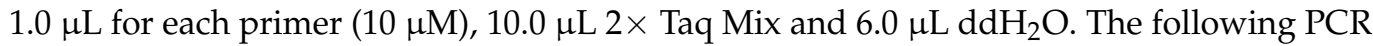
procedure is used for the amplification: $95^{\circ} \mathrm{C}$ for $5 \mathrm{~min}$, followed by 35 cycles $\left(94{ }^{\circ} \mathrm{C}\right.$ for $30 \mathrm{~s}, 55^{\circ} \mathrm{C}$ for $30 \mathrm{~s}$ and $72{ }^{\circ} \mathrm{C}$ for $1 \mathrm{~min}$ ), final extension at $72{ }^{\circ} \mathrm{C}$ for $5 \mathrm{~min}$. After PCR amplification products are detected by $1.0 \%$ agarose gel electrophoresis, target fragments are obtained by gel extraction and recycling and then are sequenced in Vazyme (Nanjing, China).

Bioinformatic analysis of target sequences is performed as described below: extrons, introns and ORFs of wheat DREB genes are analyzed with ProtParam (Expasy, Swiss), the conserved domains of amino acid sequences encoded by wheat $D R E B$ genes are analyzed by CD-search (NCBI, Bethesda, MD, USA), these homologous sequences are retrieved with BLASTP 2.12.2.0 (http:/ / blast.ncbi.nlm.nih.gov / Blast.cgi, accessed on 30 June 2021) (NCBI, Bethesda, MD, USA), and the alignments on amino acid sequences or homologous sequences of wheat DREBs are carried out using DNAMAN 6.0 (Lynnon Biosoft, San Ramon, CA, USA).

\subsection{Fluorescence Quantitative Real-Time PCR}

The expression of DREB genes in wheat is studied by qRT-PCR, the internal reference gene is $\beta$-Actin, and these primers for qRT-PCR are listed in Table S6. qRT-PCR is performed on LightCycler 96 Real-time PCR instrument, and cDNA synthesized by reverse transcription of total RNA is used as the template in qRT-PCR.

According to the instructions of AceQ qPCR SYBR Green Master Mix kit (Vazyme, Nanjing, China), qRT-PCR reaction system consists of $1.0 \mu \mathrm{L}$ AceQ qPCR SYBR Green

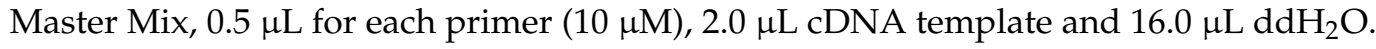
qRT-PCR is performed with the following procedures: pre-denaturation for $5 \mathrm{~min}$ at $95^{\circ} \mathrm{C}$, followed by 40 cycles of $95^{\circ} \mathrm{C}$ for $10 \mathrm{~s}$ and $60^{\circ} \mathrm{C}$ for $30 \mathrm{~s}$.

The relative expression level of wheat $D R E B$ genes under osmotic stress is normalized and analyzed by the comparative $\mathrm{Ct}\left({ }^{2-\Delta \Delta c t}\right)$ method [41]. The calculation formula is as follows: Relative expression level $=2^{-\Delta \Delta \mathrm{Ct}}, \Delta \Delta \mathrm{Ct}$ (target gene) $=\Delta \mathrm{Ct}$ (treatment group) $\Delta \mathrm{Ct}$ (control group), $\Delta \mathrm{Ct}$ (target gene) $=\mathrm{Ct}$ (target gene) $-\mathrm{Ct}$ (reference gene). Furthermore, data are obtained from three biological replicates, and each qRT-PCR experiment is repeated three times.

\subsection{Isolation and Analysis of Promoter Sequence}

The promoter regions are isolated to further analyze the expression pattern of $D R E B$ genes in wheat AK58. Specific primers are designed according to the promoter sequences 
of wheat DREB2 (GU785008), DREB6 (HG670306.1) or Wdreb2 (KF731666), and are listed in Table S6.

The target promoter sequences are amplified in $20 \mu \mathrm{L}$ PCR reaction mixture consisting of $2.0 \mu \mathrm{L}$ DNA template, $10.0 \mu \mathrm{L} 2 \times$ Taq Mix, $1.0 \mu \mathrm{L}$ for each primer $(10 \mu \mathrm{M})$ and $6.0 \mu \mathrm{L}$ $\mathrm{ddH}_{2} \mathrm{O}$. The reaction condition of PCR procedure is $95^{\circ} \mathrm{C}$ for $5 \mathrm{~min}$, followed by 40 cycles $\left(94{ }^{\circ} \mathrm{C}\right.$ for $30 \mathrm{~s}, 55{ }^{\circ} \mathrm{C}$ for $30 \mathrm{~s}$ and $72{ }^{\circ} \mathrm{C}$ for $1.5 \mathrm{~min}$ ), with the final extension at $72{ }^{\circ} \mathrm{C}$ for $5 \mathrm{~min}$. PCR amplification products are separated by $1.0 \%$ agarose gel electrophoresis. The target fragments are obtained by gel extraction and recycling, then are sequenced in Vazyme (Nanjing, China). PlantCAREand PLACE are used to analyze cis-acting elements in the promoter sequences of wheat $D R E B$ genes.

\subsection{Methylation Analysis of Promoter}

CpG islands (Island size > 100, GC Percent $>50.0$, Obs/Exp > 0.6) in the promoter regions of wheat DREBs are predicted and analyzed using EMBOSS CpG Plot (European Bioinformatics Institute, Cambridge, UK). According to the analysis of $\mathrm{CpG}$ islands, amplification primers for bisulfite sequencing PCR (BSP) are designed using MethPrimer (Chinese Academy of Medical Sciences, Beijing, China), Methyl Primer Expressv 1.0 (Applied Biosystems, Foster, CA, USA) and Primer Premier 5.0 (Premier Biosoft International, Palo Alto, Canada), and are listed in Table S5. The CpG island of DREB6 promoter is amplified in two parts (region I and region II) because of the limited length of BSP amplification.

In this study, genomic DNA from wheat leaves is firstly treated with EZ DNA Methylation-Lightning ${ }^{\mathrm{TM}}$ Kit (Zymo Research, Irvine, CA, USA), and then used as the template in BSP amplification of DREB promoters. BSP reaction system is $30 \mu \mathrm{L}$ and composed of $2.0 \mu \mathrm{L}$ bisulfite-treated DNA, $1.0 \mu \mathrm{L}$ for each primer $(10 \mu \mathrm{M}), 3.0 \mu \mathrm{L} 10 \times$ buffer $\left(\mathrm{Mg}^{2+}\right)$, 1.0 $\mu \mathrm{L}$ dNTP, $1.0 \mu \mathrm{L}$ Relia $^{\text {TM }}$ hot-start Taq polymerized aes and $21.0 \mu \mathrm{L} \mathrm{ddH}_{2} \mathrm{O}$. The PCR amplification procedure is as follows: pre-denaturation at $95^{\circ} \mathrm{C}$ for $4 \mathrm{~min}$, followed by 40 cycles $\left(94{ }^{\circ} \mathrm{C}\right.$ for $30 \mathrm{~s}, 55^{\circ} \mathrm{C}$ for $30 \mathrm{~s}, 72{ }^{\circ} \mathrm{C}$ for $\left.40 \mathrm{~s}\right)$, and a final extension at $72{ }^{\circ} \mathrm{C}$ for $5 \mathrm{~min}$. PCR amplification products are detected by $1.0 \%$ agarose gel electrophoresis. It is found that only target fragments are amplificated. The target fragments are obtained by gel extraction and recycling, and are sequenced by GENEray (Shanghai, China).

In addition, at least 10 clones are sequenced for each target fragment and three biological replicates are set up; the analysis on methylation site, methylation type and methylation rate are performed with CyMATE (Vienna University of Technology, Vienna, Austria) and Kismeth (Mount Sinai School of Medicine, New York, USA).

\subsection{Statistical Analysis}

Statistical analysis of data is performed in this study; the expression level of genes and methylation ratio of promoters are tested by significance level, ANOVA and multiple comparisons of Duncan's multiple range; the correlation between gene expression and promoter methylation is analyzed by Pearson correlation coefficient $r$ of SPSS 15.0 (SPSS, New York, USA).

\section{Conclusions}

In this study, DREB2, DREB6 and Wdreb2 are identified in wheat AK58, and one 712-bp intron is found in DREB6. Under osmotic stress, the expression patterns of DREB2, DREB6 and Wdreb2 are different, yet their expression levels are obviously higher in leaves. Some elements related to stresses are also found in the promoter regions of DREB2, DREB6 and $W d r e b 2$, while further analysis showed that promoter methylation of Wdreb2 is negatively correlated with their expression. Therefore, DREB2, DREB6 and Wdreb2 in wheat might function differently in response to osmotic stress, and promoter methylation has more significant effects on the gene expression of Wdreb2, which would be helpful to reveal the regulatory mechanism of DNA methylation in plant response to osmotic stress. 
Supplementary Materials: The following are available online at https://www.mdpi.com/article/10 $.3390 /$ ijms22147670/s1. Figure S1 The sequences of wheat DREB genes. (a), (b) and (c) represent the nucleotide sequence and amino acid sequence of DREB2, DREB6 and Wdreb2, respectively. The box and underline denote nuclear localization signal and AP2/EREBP domain, respectively. Figure S2 Alignments of wheat DREBs. (a) and (b) represent the alignments of AP2/EREBP domain and amino acid sequence of DREB2, DREB6 and Wdreb2. The similar and consistent amino acids are indicated in blue or dark blue, respectively. $\beta$-sheet and $\alpha$-helix are indicated with long arrow and dotted line, and the conserved residues at 14th and 19th are marked with short arrow. Figure S3 Multiple alignment of wheat DREBs and its homologous sequences. (a), (b) and (c) are the alignment on some homologous sequences of wheat DREB2, DREB6 and Wdreb2, respectively. The underline denotes the AP2/EREBP domain. Figure S4 Promoter regions of wheat DREB genes. (a), (b) and (c) represent the promoter sequences and some cis-acting elements of DREB2, DREB6 and Wdreb2, respectively. Figure S5 Prediction of $\mathrm{CPG}$ islands in the promoter regions of wheat DREB genes. (a), (b) and (c) represent the location of putative CPG islands in the promoter regions of DREB2, $D R E B 6$ and Wdreb2, respectively. The CPG island regions between two arrows below each diagram were examined using BSP. Figure S6 Bisulfite sequencing analysis on the promoter regions of wheat $D R E B$ genes using CyMATE. (a), (b) and (c) indicate methylation sites in the promoter regions of $D R E B 2, D R E B 6$ and Wdreb2 in wheat leaves. Circles, squares and triangles represent CG, CHG or $\mathrm{CHH}$; filled and hollow circles denote methylated and unmethylated cytosine. Each row represents the sequencing result of one positive clone. The length of $\mathrm{CpG}$ island sequence is visualized above each diagram and the potential methylation sites are displayed below. Table S1 Homologous amino acid sequences of wheat DREBs by BLASP. The homologous sequences of wheat DREB2, DREB6 and Wdreb2 were retrieved with BlastP algorithm, which are mainly from some species in Gramineae. The homologous sequences having higher identity with wheat DREBs were selected from some Genera in Gramineae and were further analyzed. Table S2 The cis-acting elements in the promoter of DREB2. Table S3 The cis-acting elements in the promoter of DREB6. Table S4 The cis-acting elements in the promoter of Wdreb2. Table S5 Correlation between promoter methylation and expression of $D R E B$ genes. Pearson's r represents the relationship between expression levels of DREB2, DREB6, Wdreb2 in wheat leaves and methylation rates at CG, $\mathrm{CHG}$ or $\mathrm{CHH}$ sites in their promoter regions under the treatment of $15 \% \mathrm{PEG}_{6000}$ solution. According to the expression level and methylation rate of wheat seedlings incubated in $\mathrm{PEG}_{6000}$ solution for $12 \mathrm{~h}$, Pearson's $\mathrm{r}$ analysis of DREBs were performed. Table S6 Primers used in this study.

Author Contributions: H.D. and Y.Z. conceived this experiment; H.W., P.Y. and S.S. analyzed experiment data and wrote this paper; P.C., Z.D. and T.D. participated in text editing; L.J., L.L. and H.D. revised this manuscript. All authors have read and agreed to the published version of the manuscript.

Funding: This research was completed under the financial aid of National Science-technology Support Plan Projects (2013BAD07B14), National Science Foundation of China (31870312) and Fund of Henan Normal University (2019JQ01) in China.

Data Availability Statement: The data presented in the current study are available in the article and supplementary materials.

Conflicts of Interest: The authors declare that they have no competing interests.

\section{Abbreviations}

BSP bisulfite sequencing PCR

CTAB cetyltriethyl ammonium bromide

DRE/CRT dehydration responsive element/C-repeat

DREB dehydration responsive element binding protein

qRT-PCR quantitative real-time PCR 


\section{References}

1. Reis, R.R.; Cunha, B.A.D.; Martins, P.K.; Martins, M.T.B.; Alekcevetch, J.C.; Chalfun-Junior, A.; Andrade, A.C.; Ribeiro, A.P.; Qin, F.; Mizoi, J.; et al. Induced over-expression of AtDREB2A CA improves drought tolerance in sugarcane. Plant Sci. 2014, 221, 59-68. [CrossRef]

2. Lipiec, J.; Doussan, C.; Nosalewicz, A.; Kondracka, K. Effect of drought and heat stresses on plant growth and yield: A review. Int. Agrophys. 2013, 27, 463-477. [CrossRef]

3. Wang, P.; Du, Y.; Zhao, X.; Miao, Y.; Song, C.P. The MPK6-ERF6-ROS-responsive cis-acting element7/GCC box complex modulates oxidative gene transcription and the oxidative response in Arabidopsis. Plant Physiol. 2013, 161, 1392-1408. [CrossRef] [PubMed]

4. Huang, S.C.; Chu, S.J.; Guo, Y.M.; Ji, Y.J.; Hu, D.Q.; Cheng, J.; Lu, G.H.; Yang, R.W.; Tang, C.Y.; Qi, J.L.; et al. Novel mechanisms for organic acid-mediated aluminium tolerance in roots and leaves of two contrasting soybean genotypes. AoB Plants 2017, 9 , plx064. [CrossRef] [PubMed]

5. Yu, M.; Liu, H.J.; Dong, Z.Y.; Xiao, J.W.; Su, B.D.; Fan, L.S.; Kmis, G.; Samaj, J.; Lin, J.X.; Li, R.L. The dynamics and endocytosis of Flot1 protein in response to flg22 in Arabidopsis. J. Plant Physiol. 2017, 215, 73-84. [CrossRef] [PubMed]

6. Sazegari, S.; Niazi, A.; Ahmadi, F.S. A study on the regulatory network with promoter analysis for Arabidopsis DREB genes. Bioinformation 2015, 11, 101-106. [CrossRef] [PubMed]

7. Sakuma, Y.; Maruyama, K.; Osakabe, Y.; Qin, F.; Seki, M.; Shinozaki, K.; Yamaguchi-Shinozaki, K. Functional analysis of an Arabidopsis transcription factor, DREB2A, involved in drought-responsive gene expression. Plant Cell 2006, 18, 1292-1309. [CrossRef]

8. Jaglo-Ottosen, K.R.; Gilmour, S.J.; Zarka, D.G.; Schabenberger, O.; Thomashow, M.F. Arabidopsis CBF1 overexpression induces cor genes and enhances freezing tolerance. Science 1998, 280, 104-106. [CrossRef] [PubMed]

9. Liu, Q.; Kasuga, M.; Sakuma, Y.; Abe, H.; Miura, S.; Yamaguchi-Shinozaki, K.; Shinozaki, K. Two transcription factors, DREB1 and DREB2, with an EREBP/AP2 DNA binding domain separate two cellular signal transduction pathways in drought- and low-temperature-responsive gene expression, respectively, in Arabidopsis. Plant Cell 1998, 10, 1391-1406. [CrossRef] [PubMed]

10. Dossa, K.; Wei, X.; Li, D.; Fonceka, D.; Zhang, Y.X.; Wang, L.H.; Yu, J.Y.; Boshou, L.; Diouf, D.; Cisse, N.; et al. Insight into the AP2/ERF transcription factor superfamily in sesame and expression profiling of DREB subfamily under drought stress. $B M C$ Plant Boil. 2016, 16, 171. [CrossRef]

11. Gehring, M.; Henikoff, S. DNA methylation dynamics in plant genomes. Biochim. Biophys. Acta 2007, 1769, 276-286. [CrossRef] [PubMed]

12. Duan, H.Y.; Liu, W.X.; Li, J.Y.; Ding, W.K.; Zhu, Y.Q.; Wang, H.N.; Jiang, L.N.; Zhou, Y.Q. Growth and DNA methylation level of Triticum aestivum seedlings treated with 5-azacytidine. Pak. J. Bot. 2016, 48, 1585-1591.

13. Zheng, X.G.; Chen, L.; Lou, Q.J.; Li, M.S.; Luo, L.J. Changes in DNA methylation pattern in a water-saving and drought-resistance rice variety at three-leaf and four-leaf stages after drought domestication. Chin. J. Rice Sci. 2014, 28, 32-40.

14. Fan, H.H.; Li, T.C.; Li, Z.P.; Lin, Y.; Cai, Y.P.; Jin, Q. MSAP analysis of epigenetic changes in Dendrobium huoshanense under PEG simulated drought stress. Acta Agric. Nucleatae Sin. 2011, 25, 363-368.

15. Tang, X.M.; Tao, X.; Wang, Y.; Ma, D.W.; Li, D.; Yang, H.; Ma, X.R. Analysis of DNA methylation of perennial ryegrass under drought using the methylation-sensitive amplification polymorphism (MSAP) technique. Mol. Genet. Genom. 2014, 289, 1075-1084. [CrossRef]

16. Chinnusamy, V.; Zhu, J. Epigenetic regulation of stress responses in plants. Curr. Opin. Plant Biol. 2009, 12, 133-139. [CrossRef]

17. Zeng, F.S. Drought resistance and DNA methylation of interspecific hybrids between Fraxinus mandshurica and Fraxinu samericana. Trees 2014, 28, 1679-1692. [CrossRef]

18. Yang, M.N.; Yang, G.L.; Guo, T.; Liu, Y.Z.; Zhang, J.G.; Chen, Z.Q.; Wang, H. DNA methylation under stresses and its prospects in plant drought-resistant breeding. Chin. Agric. Sci. Bull. 2013, 6, 6-11.

19. Zhang, C.Y. Study on the Genetic Epigenetic Variation and Related Physiological Metabolism Changes in Rice under Drought Stress; Northeast Normal University: Changchun, China, 2013.

20. Ahmed, M.D.; Khan, A.S.; Kashif, M.; Khan, S. Genetic mechanism of leaf venation and stomatal traits for breeding drought tolerant lines in wheat. Bangladesh J. Bot. 2017, 46, 35-41.

21. Gupta, P.; Balyan, H.S.; Gahlaut, V. QTL analysis for drought tolerance in wheat: Present status and future possibilities. Agronomy 2017, 7, 5. [CrossRef]

22. Ni, Z.Y.; Xu, Z.S.; Liu, L.; Li, L.C.; Chai, Y.; Chen, M.; Ma, Y.Z. Isolation and characterization of a transcription factor TaDREB6 gene from Triticum aestivum L. J. Triticeae Crop. 2008, 28, 357-363.

23. Pan, L. Excavation of Natural Allelic Variation of Wheat DREB2 Transcription Factor; University of Electronic Science and Technology of China: Chengdu, China, 2010.

24. Dubouzet, J.G.; Sakuma, Y.; Ito, Y.; Kasuga, M.; Dubouzet, E.G.; Miura, S.; Seki, M.; Shinozaki, K.; Yamaguchi-Shinozaki, K. OsDREB genes in rice (Oryza sativa L.) encode transcription activators that function in drought-, high-salt- and cold-responsive gene expression. Plant J. 2003, 33, 751. [CrossRef] [PubMed]

25. Li, L.B.; Zhao, H.; Liu, L.; He, C.F.; Bai, R.; Wang, T. Isolation and characterisation of a cold-induced DREB gene from Cymbidium insigne. J. Hortic. Sci. Biotechnol. 2011, 86, 43-49. [CrossRef]

26. Lopato, S.; Bazanova, N.; Morran, S.; Milligan, A.S.; Shirley, N.; Langridge, P. Isolation of plant transcription factors using a modified yeast one-hybrid system. Plant Methods 2006, 2, 45-66. [CrossRef] 
27. Liang, C.B. Cloning and expressing analysis of a DREB transcription factor in Sunflower. Ningxia J. Agric. For. Sci. Technol. 2015, 1, 31-34.

28. Huang, Y.; Xu, Z.S.; Wang, F.; Song, X.; Tian, C.; Xiong, A.S. Isolation and expression profiles analysis of two Dc DREB-A1 group transcription factor genes from Carrot. J. Nucl. Agric. Sci. 2015, 29, 29-39.

29. Fang, Z.W.; Xu, X.Y.; Gao, J.F.; Wang, P.K.; Liu, Z.X.; Feng, B.L. Characterization of FeDREB1 promoter involved in cold- and drought-inducible expression from common buckwheat (Fagopyrum esculentum). Genet. Mol. Res. 2015, 14, 7990-8000. [CrossRef]

30. Sreenivasulu, G.; Senthilkumaran, B.; Sudhakumari, C.C.; Guan, G.; Oba, Y.; Kagawac, H.; Nagahama, Y. 20ß-hydroxysteroid dehydrogenase gene promoter: Potential role for cyclic AMP and xenobiotic responsive elements. Gene 2012, 509, 68-76. [CrossRef]

31. Jones, P.A. Functions of DNA methylation: Islands, start sites, gene bodies and beyond. Nat. Rev. Genet. 2012, 13, 484-492. [CrossRef]

32. Abid, G.; Mingeot, D.; Muhovski, Y.; Mergeai, G.; Aouida, M.; Abdelkarim, S.; Aroua, I.; Ayed, M.E.; Mhamdi, M.; Sassif, K. Analysis of DNA methylation patterns associated with drought stress response in faba bean (Vicia faba L.) using methylationsensitive amplification polymorphism (MSAP). Environ. Exp. Bot. 2017, 142, 34-44. [CrossRef]

33. Duan, H.Y.; Li, J.Y.; Zhu, Y.Q.; Jia, W.J.; Wang, H.H.; Jiang, L.N.; Zhou, Y.Q. Responsive changes of DNA methylation in wheat (Triticum aestivum) under water deficit. Sci. Rep. 2020, 10, 7938. [CrossRef]

34. Kumar, S.; Singh, A. Epigenetic regulation of abiotic stress tolerance in plants. Adv. Plants Agric. Res. 2016, 5, 179. [CrossRef]

35. Zilberman, D.; Gehring, M.; Tran, R.; Ballinger, T.; Henikoff, S. Genome-wide analysis of Arabidopsis thaliana DNA methylation uncovers an interdependence between methylation and transcription. Nat. Genet. 2007, 39, 61-69. [CrossRef] [PubMed]

36. Song, Y.G.; Ma, Z.Q.; Qiu, N.W.; Dong, W. DNA methylation modification status of CUC1 during in vitro organogenesis in Arabidopsis. Plant Physiol. J. 2016, 6, 926-932.

37. Wang, R.X. Research of Transcriptional Regulation of Opaque2 Gene and DNA Methylation of 19-kDa $\alpha$-zeins in Maize; Zhejiang University: Hangzhou, China, 2015.

38. Zhang, X.; Yazaki, J.; Sundaresan, A.; Cokus, S.; Chan, A.; Chen, H.M.; Henderson, I.R.; Shin, P.; Pellegrini, M.; Jacobsen, S.E.; et al. Genome-wide high-resolution mapping and functional analysis of DNA methylation in Arabidopsis. Cell 2006, 126, $1189-1201$. [CrossRef] [PubMed]

39. Yang, W.; Pan, Y.; Su, C.G. Analysis of the DNA methylation of SlGLD1 regulated by JMJ524 in tomato. Biotechnol. Bull. 2016, 32, 79-85.

40. Wang, H.N.; Li, B.Y.; Chen, F.F.; Zhou, Y.Q.; Li, J.J.; Zheng, X.; Duan, H.Y. Analysis on phenotype, catalpol accumulation and methylation of Rehmannia glutinosa. Pak. J. Bot. 2019, 51, 461-467. [CrossRef]

41. Duan, H.Y.; Liu, W.X.; Zeng, Y.P.; Jia, W.J.; Wang, H.H.; Zhou, Y.Q. Expression analysis of key enzymes involved in the accumulation of iridoid in Rehmannia glutinosa. Plant Omics 2019, 12, 102-108. [CrossRef] 